\title{
Intra-ligamentous fibroid removed laparoscopically
}

\author{
Richard L. Heaton*, M. Sami Walid
}

Heart of Georgia Women's Center, Warner Robins, USA.

E-mail: *riclheaton@yahoo.com

Received 25 July 2011; revised 18 August 2011; accepted 25 August 2011.

\begin{abstract}
In this paper, we report the case of a left round ligament fibroid in a 24 year-old, gravida 0 , female that was successfully managed laparoscopically. Operative findings also included severe adnexal adhesions bilaterally, severe adhesions of the liver to the anterior abdominal wall (Fitz-Hugh-Curtis syndrome), pigmented areas on the left tube, uterus and posterior broad ligament (that proved to be endometriosis), and low capacity bladder $(450 \mathrm{cc}$ at $70 \mathrm{~cm}$ water pressure) with florid glomerulations and Hunner's ulcers consistent with typical interstitial cystitis. Beside the feasibility of laparoscopic management of intra-ligamentous tumors this case highlights the common multifactorial nature of chronic pelvic pain and the frequent association of endometriosis and interstitial cystitis.
\end{abstract}

Keywords: Intra-Ligamentous; Round Ligament; Fibroid; Leiomyoma; Pelvic Pain; Endometriosis; Interstitial Cystitis

\section{INTRODUCTION}

The round ligament of the uterus may rarely become the site of oncologic formation, the most frequent histologic type of which is leiomyoma [1]. Intra-round-ligament fibroid may grow at any point along the track of the ligament, from the uterine horns to the entry opening of the inguinal canal, in the inguinal canal, or after exiting the inguinal canal in the labia majora or mons pubis mimicking therefore other mass lesions in the region such as adenopathy or inguinal hernias [1-4]. Unlike other leiomyomas, intra-ligamentous tumors can grow in already hysterectomised patients [5]. In this paper, we report a case of round ligament fibroid that was successfully managed using a minimal invasive route.

\section{CASE REPORT}

A 24 year-old, gravida 0, $127 \mathrm{lb} ., 5 \mathrm{ft} .2$ in., female presented with complaints of chronic socially-compro- mising bleeding with right-sided pelvic pain. She had elevated Pelvic Pain and Urgency/Frequency (PUF) score of 10, positive potassium sensitivity test (PST) and positive anesthetic challenge test. On ultrasound, the left ovary was normal but the right ovary appeared displaced behind the uterus. There was slight endometrial hypertrophy (sounding to $8 \mathrm{~cm}$ ).

The patient underwent diagnostic and operative laparoscopy, hysteroscopy, and cystoscopy with hydrodistension. Operative findings were severe adnexal adhesions bilaterally, severe adhesions of the liver to the anterior abdominal wall (Fitz-Hugh-Curtis syndrome, Figure 1), a tumor from the left round ligament (Figure 2), pigmented areas on the left tube, uterus and posterior broad ligament, and low capacity bladder (450 cc at $70 \mathrm{~cm}$ water pressure) with florid glomerulations and Hunner's ulcers consistent with typical interstitial cystitis (Figure 3).

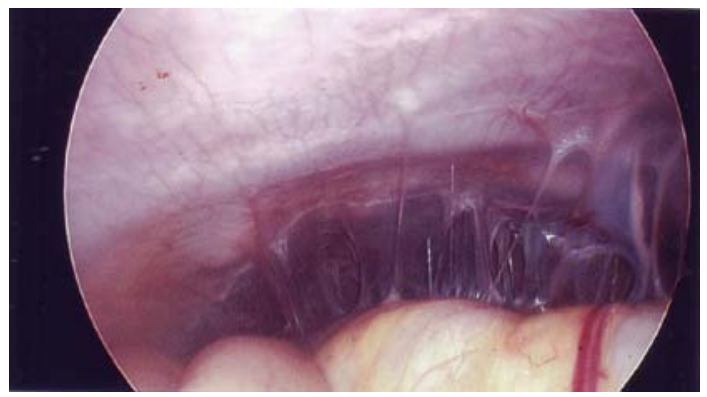

Figure 1. Perihepatic adhesions consistent with FitzHugh-Curtis syndrome.

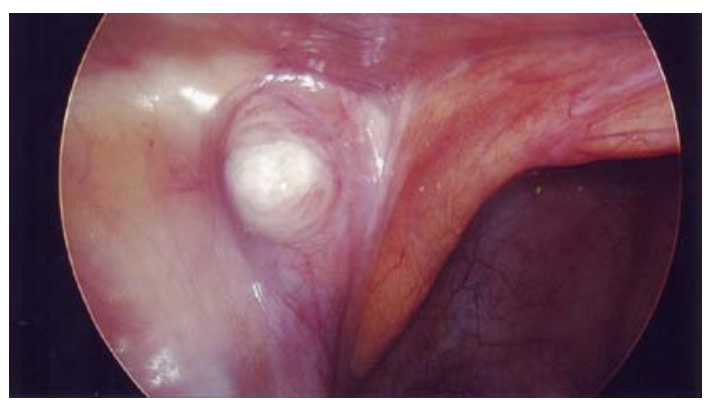

Figure 2. Leiomyoma at the anteriolateral left junction of the round ligament with the peritoneum. 


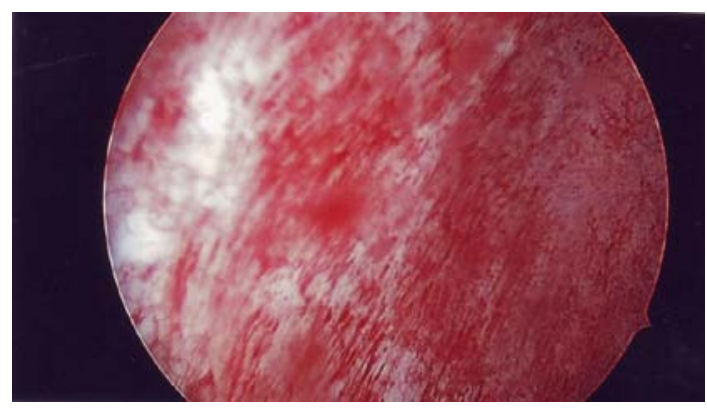

Figure 3. Florid glomerulations and Hunner's ulcers of the bladder consistent with typical interstitial cystitis.

Extensive lysis of adhesions, biopsying of pigmented areas and laparoscopic excision of the round ligament tumor were performed. Hysteroscopically, both cornua appeared to be normal and open; there was no evidence of submucosal pigmentations consistent with adenomyosis. Pathologic report showed endometriosis and the round ligament tumor proved to be a leiomyoma. Operative time was 65 minutes and blood loss was estimated at 100 cc.

\section{LAPAROSCOPIC TECHNIQUE}

The leiomyomatous tumor was located at the anteriolateral left junction of the round ligament with the peritoneum. Laparoscopic removal of interligementous fibroids is accomplished by traction counter traction with either sharp or harmonic dissection taking care to coagulate any bleeding sites as encountered, to maintain a dry field and optimal visualization to avoid injury to surrounding structures. Removal of a tumor, if small, can be done with an endopouch, and if large, morcellation is required.

\section{COMMENTARY}

Intra-ligamentous fibroids affecting the round ligament are not common. During a busy gynsurgical career we have seen intraligamentous fibroids involving the labia majora, the lower third of the right lateral vaginal wall, and the broad ligament of the uterus. It is important to remember that a solid tumor anywhere in the pelvis may be a fibroid. These misplaced tumors usually lead to exploratory surgery to rule out more sinister lesions. Since fibroids are hormone-sensitive they tend to grow until menopause or until they outgrow their blood supply. Therefore when seen during diagnostic laparoscopy it is our recommendation to remove them and prevent a subsequent exploratory surgery.

The other interesting aspect of this case is the coexistent endometriosis and interstitial cystitis. We have previously reported that in our retrospective series there was a $60 \%$ comorbid state with endometriosis or adenomyosis when Interstitial cystitis (IC) is present [6]. We are disturbed by the de-emphasis of the importance of cystoscopy with hydrodistension in the evaluation of possible IC. With such a high rate of comorbid disease, if the patient is already under anesthesia for evaluation of pelvic pain laparoscopically why would one pass up the opportunity to diagnose a frequent comorbid disease and get prognostic information based on anesthetic bladder capacity? As it appears that prognosis for remission is good with normal bladder capacity and progressively worsens with loss of bladder capacity [7]. Also since the symptom complex for adenomyosis and endometriosis overlap and adenomyosis can be confirmed but not excluded hysteroscopically it seems somewhat negligent not to hysteroscope these patients. The senior author (Dr. Heaton) in the past used a resectoscope to biopsy pigmented submucosal uterine lesions; the pathology uniformally revealed ademomyosis. Endometriosis, adenomyosis and leiomyomata develop in women of reproductive age and regress after menopause or removal of ovaries, indicating that they grow in an estrogen-dependent manner. These diseases appear to have some common pathogenetic pathways [8]. A recent study suggested that the PVUII polymorphism of the ERalpha gene is associated with the risk for endometriosis, adenomyosis, and leiomyomata [9].

We have reported previously on the common multifactorial nature of chronic pelvic pain [10]. This patient is yet another example of this concept. It is our opinion that if a patient has enough cyclic pelvic pain to indicate laparoscopy then hysteroscopy is warranted as well to evaluate for possible adenomyosis. If endometriosis or adenomyosis or an elevated PUF score is present it is also our opinion that with the patient already asleep for laparoscopy failing to take the opportunity to confirm the diagnosis of IC and obtain prognostic information seems to be less than optimal care especially with the patients having a $50 \%$ chance of temporary relieve of IC symptoms for several months after hydrodistension $[7,11]$.

\section{REFERENCES}

[1] Michel, P. and Viola, D. (2003) Abdomino-pelvic leiomyoma of the round ligament: Contribution of computed tomography and magnetic resonance imaging. Journal of Gynecology and Obstetric Biology Reproductive, 32, 571574.

[2] Vignali, M., Bertulessi, C., Spreafico, C. and Busacca, M. (2006) A large symptomatic leiomyoma of the round ligament. Minimally Invasive Gynecology, 13, 375-376. doi:10.1016/j.jmig.2006.03.008

[3] Grossman, S.L. and Cheung, V.Y. (1999) Round ligament fibroid. Journal of Obstetrics and Gynaecology of Canadian, 29, 109-110.

[4] Warshauer, D.M. and Mandel, S.R. (1999) Leiomyoma of the extra-peritoneal round ligament: CT demonstration. Clinical Imaging, 23, 375-376. 
doi:10.1016/S0899-7071(98)00021-7

[5] Lösch, A., Haider-Angeler, M.G., Kainz, C., Breitenecker, G. and Lahodny, J. (1999) Leiomyoma of the round ligament in a post-menopausal woman. Maturitas, 31, 133135.

[6] Walid, M.S. and Heaton, R.L. (2010) Endometriosis/ adenomyosis is associated with more typical cystoscopic findings of interstitial cystitis in patients with elevated PUF scores. Gynecological Surgery, 7, 353-357. doi:10.1007/s10397-010-0587-y

[7] Glemain, P., Rivière, C., Lenormand, L., Karam, G., Bouchot, O. and Buzelin, J.M. (2002) Prolonged hydrodistention of the bladder for symptomatic treatment of interstitial cystitis: Efficacy at 6 months and 1 year. European Urology, 41, 79-84. doi:10.1016/S0302-2838(01)00006-9

[8] Uimari, O., Järvelä, I. and Ryynänen, M. (2011) Do symptomatic endometriosis and uterine fibroids appear together? Journal of Human Reproductive Science, 4, 34-38. doi:10.4103/0974-1208.82358

[9] Kitawaki, J., Obayashi, H., Ishihara, H., Koshiba, H., Kusuki, I., Kado, N., Tsukamoto, K., Hasegawa, G., Nakamura, N. and Honjo, H. (2001) Oestrogen receptoralpha gene polymorphism is associated with endometriosis, adenomyosis and leiomyomata. Human Reproductive, 16, 51-55. doi:10.1093/humrep/16.1.51

[10] Walid, M.S. and Heaton, R.L. (2009) Dyspareunia: A complex problem requiring a selective approach. Sex Health, 6, 250-253. doi:10.1071/SH09033

[11] Rigaud, J., Delavierre, D., Sibert, L. and Labat, J.J. (2010) Hydrodistension in the therapeutic management of painful bladder syndrome. Prog Urology, 20, 1054-1059. doi:10.1016/j.purol.2010.08.049 